\title{
End-to-end Quality of Service in Pseudo-Wire networks
}

\author{
Mohamed Lamine Lamali \\ Alcatel-Lucent Bell Labs \\ France \\ mohamed_lamine.lamali@alcatel- \\ lucent.com
}

\author{
Helia Pouyllau \\ Alcatel-Lucent Bell Labs \\ France \\ helia.pouyllau@alcatel- \\ lucent.com
}

\author{
Dominique Barth \\ Lab. PRiSM, UMR8144 \\ University of Versailles \\ dominique.barth@prism.uvsq.fr
}

\section{INTRODUCTION}

Carrier-grade networks are complex systems that include several heterogeneous domains and support various types of services under specific Quality of Service (QoS) requirements. To tackle the problem of setting end-to-end connections across heterogeneous domains, the Pseudo-Wire architecture [1] allows to emulate some protocols (e.g. SDH, Ethernet, ATM, etc.) over MPLS. This emulation is achieved by encapsulation and decapsulation functions called adaptation functions. A path crossing heterogeneous domains must involve compatible functions so that datagrams are understandable by the source and target nodes (e.g. if Ethernet is encapsulated in MPLS by a node, it must be decapsulated by another).

When crossing several carriers, this heterogeneity issue becomes more pressing in order to support future collaborative inter-domain services. However, to support such services, the path computation problem has been mostly addressed under end-to-end (e2e) QoS requirements (e.g. delay $\leq 100 \mathrm{~ms}$ ). In [2], the authors present algorithms solving this problem. To the best of our knowledge, the compatibility of adaptation functions has not been yet considered together with e2e QoS constraints.

This paper focuses on the computation of "feasible" (i.e. involving compatible adaptation functions) paths under QoS constraints. Section 2 is a first step towards the description of this problem and its complexity and section 3 sketches the possible approaches to solve it.

\footnotetext{
${ }^{*}$ This work is partially supported by the ETICS-project, funded by the European Commission. Grant agreement no.: FP7-248567 Contract Number: INFSO-ICT-248567.
}

Permission to make digital or hard copies of all or part of this work for personal or classroom use is granted without fee provided that copies are not made or distributed for profit or commercial advantage and that copies bear this notice and the full citation on the first page. To copy otherwise, to republish, to post on servers or to redistribute to lists, requires prior specific permission and/or a fee.

ACM CoNEXT Student Workshop, December 6, 2011, Tokyo, Japan.

Copyright 2011 ACM 978-1-4503-1042-0/11/0012 ...\$10.00.

\section{E2E QOS IN PSEUDO-WIRE NETWORKS}

\subsection{Pseudo-wire network model}

Consider a network with a set of nodes $V$, a set of links $E$ and a set of protocols $A$. Each node $v$ is associated to a set $\mathcal{Q}_{v}$ of $\mathrm{QoS}$ vectors whose components are QoS parameter values, a set of supported protocols $A_{v}$ and a set of adaptation functions $F_{v}$. Let two protocols $a_{m}, a_{n} \in A,\left(a_{m} \rightarrow a_{n}\right) \in F_{v}$ and $\left(a_{m} \leftarrow a_{n}\right) \in F_{v}$ express respectively the encapsulation of $a_{m}$ in $a_{n}$ and decapsulation of $a_{m}$ from $a_{n} .\left(a_{m} \rightarrow a_{m}\right) \in F_{v}$ denotes that $a_{m}$ passively crosses $v$. In addition, we denote by $z\left(v, a_{m} \rightarrow a_{n}, q_{v}\right)$ the price of using the adaptation function $\left(a_{m} \rightarrow a_{n}\right)$ and the QoS vector $q_{v}$.

Feasible path. The goal is to compute a path from $v_{0}$ to $v_{t}$ which involves compatible adaptation functions and meets QoS requirements. A path is a sequence of triples in the form $\left(v, q_{v}, a_{v}\right)$, where $v \in V, q_{v} \in \mathcal{Q}_{v}$ is the chosen QoS vector, and $a_{v} \in A_{v}$ is the chosen protocol to cross the node $v$.

Let $\left(v_{0}, q_{v_{0}}, a_{0}\right),\left(v_{1}, q_{v_{1}}, a_{1}\right),\left(v_{2}, q_{v_{2}}, a_{2}\right), \ldots$, $\left(v_{m}, q_{v_{m}}, a_{m}\right),\left(v_{t}, q_{v_{t}}, a_{m+1}\right)$ be a path. This path is feasible if $i$ ) $\left.a_{0}=a_{m}, i i\right)$ each encapsulated protocol has been decapsulated before reaching $v_{t}$, and iii) $a_{k} \in A_{v_{k}}$ for each $k \leq m$ and $\left(a_{k} \rightarrow a_{k+1}\right) \in F_{v_{k}}$ or $\left(a_{k+1} \leftarrow\right.$ $\left.a_{k}\right) \in F_{v_{k}}$ for each $k \leq m$.

QoS vectors. Given a $\mathrm{QoS}$ vector $q_{v}$ of a node $v$, we denote by $B\left(q_{v}\right), D\left(q_{v}\right)$ and $L\left(q_{v}\right)$ the bandwidth, the delay and the packet loss respectively. Note that, further QoS parameters could be considered but these parameters represent all the possible mathematical types since bandwidth is concave, delay is additive and packet loss is multiplicative.

\subsection{Problem definition}

Given a path $P_{v_{0}, v_{t}}$, the objective function, denoted $Z\left(P_{v_{0}, v_{t}}\right)$, is the sum of the prices of the selected adaptation functions and QoS vectors of the involved nodes. QoS constraints are formulated over e2e QoS requirements $B_{\min }, D_{\max }$ and $L_{\max }$. 


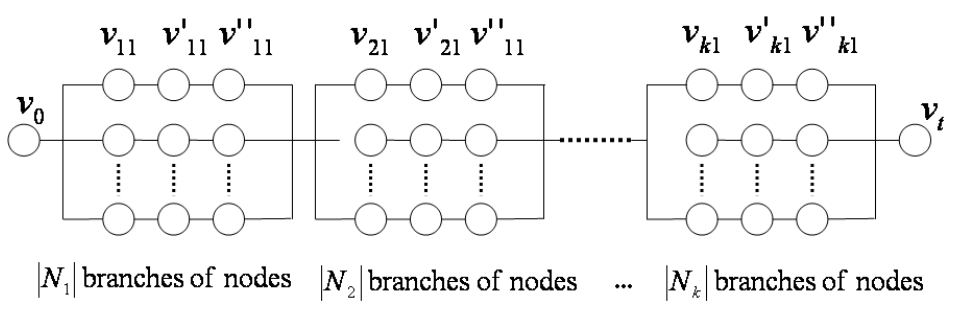

Figure 1: A series-parallel network built by reduction from the 2DMKP

$$
\begin{aligned}
& \operatorname{minimize} Z\left(P_{v_{0}, v_{t}}\right) \\
& \text { s.t. }\left\{\begin{array}{l}
P_{v_{0}, v_{t}} \text { is a feasible path } \\
\min _{q_{v} \in P_{v_{0}, v_{t}}} B\left(q_{v}\right) \geq B_{\min } \\
\sum_{q_{v} \in P_{v_{0}, v_{t}}} D\left(q_{v}\right) \leq D_{\max } \\
\prod_{q_{v} \in P_{v_{0}, v_{t}}}\left(1-L\left(q_{v}\right)\right) \geq 1-L_{\max }
\end{array}\right.
\end{aligned}
$$

In the decision version of problem (1) minimize $Z\left(P_{v_{0}, v_{t}}\right)$ is replaced by $Z\left(P_{v_{0}, v_{t}}\right) \leq Z_{\max }$.

\subsection{Complexity}

The decision version of the problem (1) is in $N P$ : the compliance of a path with the QoS, feasibility and price constraints can be checked in polynomial time. To prove that the problem (1) is $N P$-hard, we provide a polynomial reduction from the decision minimization version of the 2-Dimensional Multiple choice Knapsack Problem (2DMKP) as formalized by (2) to the decision version of the problem (1), as the minimization version of the 2DMKP is known to be NP-Complete [3].

$$
\begin{aligned}
& \sum_{i=1}^{k} \sum_{j \in N_{i}} p_{i j} x_{i j} \leq M \\
\text { s.t. } & \left\{\begin{array}{l}
\sum_{i=1}^{k} \sum_{j \in N_{i}} w_{i j} x_{i j} \leq c \\
\sum_{i=1}^{k} \sum_{j \in N_{i}} w_{i j}^{\prime} x_{i j} \leq c^{\prime} \\
\sum_{j \in N_{i}} x_{i j}=1, x_{i j} \in\{0,1\} \\
x_{i j} \in\{0,1\}, i=1, \ldots, k \quad j \in N_{i}
\end{array}\right.
\end{aligned}
$$

Sketch of proof. Figure 1 is an illustration of a seriesparallel network obtained from an instance of the problem (2) as follows: in order to represent possible adaptation functions for 2 given protocols $a_{1}$ and $a_{2}$, each object $x_{i j}$ is transformed into 3 nodes $v_{i j}, v_{i j}^{\prime}$ and $v_{i j}^{\prime \prime}$ in series, s.t. $F_{v_{i j}}=\left\{\left(a_{1} \rightarrow a_{2}\right)\right\}, F_{v_{i j}^{\prime}}=\left\{\left(a_{2} \rightarrow a_{2}\right)\right\}$ and $F_{v_{i j}^{\prime \prime}}=\left\{\left(a_{1} \leftarrow a_{2}\right)\right\}$. QoS vectors are obtained s.t. $\mathcal{Q}_{v_{i j}}=\mathcal{Q}_{v_{i j}^{\prime}}=\mathcal{Q}_{v_{i j}^{\prime \prime}}=\left\{q_{i j}\right\}$, where $D\left(q_{i j}\right)=w_{i j} / 3$ and $L\left(q_{i j}\right)=1-\exp ^{-w_{i j}^{\prime} / 3}$; and the prices are s.t. $z\left(v_{i j}, a_{1} \rightarrow\right.$ $\left.a_{2}, q_{i j}\right)=z\left(v_{i j}^{\prime}, a_{2} \rightarrow a_{2}, q_{i j}\right)=z\left(v_{i j}^{\prime \prime}, a_{1} \leftarrow a_{2}, q_{i j}\right)=p_{i j} / 3$.

The sets of 3 nodes corresponding to objects in the same class $N_{i}$ are disposed in parallel. Thus, each class $N_{i}$ is transformed into $\left|N_{i}\right|$ sets of 3 nodes in parallel and the parts of the network corresponding to each class are disposed in series. The nodes $v_{0}$ and $v_{t}$ are identified as the source and target nodes.

Let $Z_{\max }=M, D_{\max }=c$ and $L_{\max }=1-\exp ^{-c^{\prime}}$, the reduction described above is linear in the parameters of the problem (2), and the problem obtained by this transformation, which is a a subset of instances of the decision version of the problem (1), is satisfied if and only if the problem (2) is satisfied.

\section{RESEARCH AGENDA}

To check the existence and compute a feasible path under QoS constraints, we will explore two approaches. First, we expect to design a dynamic programming algorithm. However, the efficiency of such an algorithm depends strongly on the set of adaptation functions of each node. The "feasibility" of a path does not exhibit an optimal structure in the general case. For example, considering three nodes $v_{0}, v_{1}$ and $v_{2}$, it is possible to create an instance of the problem (1) in which there are no feasible paths between $v_{0}$ and $v_{1}$ and between $v_{1}$ and $v_{2}$, but there is a feasible path between $v_{0}$ and $v_{2}$ via $v_{1}$. Thus, a classification of the instances of the problem (1) should be done to characterize the subset of instances on which a dynamic programing algorithm is efficient. In another approach, we plan to design general heuristics including meta-heuristics. Ant colony is naturally adapted to compute paths under constraints, the main challenge lies in the modeling of the path "feasibility".

In order to evaluate the behavior and the robustness of the designed algorithms, we aim to conceive a simulator capable of generating network topologies with realistic patterns of QoS and adaptation function distributions.

\section{REFERENCES}

[1] S. Bryant and P. Pate. RFC3985 - Pseudo Wire Emulation Edge-to-Edge Architecture, 2005.

[2] Z. Wang and J. Crowcroft. Quality-of-Service Routing for Supporting Multimedia Applications. IEEE Journal on Selected Areas in Communications, 14(7):1228-1234, 1996.

[3] H. Kellerer, U. Pferschy, and D. Pisinger. Knapsack problems. Springer, 2004. 